Note: This paper is not the copy of record and may not exactly replicate the authorative document published in Evidence-Based Practice in Child and Adolescent Mental Health. The final article is available at doi: 10.1080/23794925.2022.2025629.

Strategies for incorporating culture into psychosocial interventions for youth of color

Alayna L. Park, Ph.D.

Palo Alto University

Leslie R. Rith-Najarian, Ph.D.

Harvard University

Dana Saifan, M.A., C. Phil., Resham Gellatly, Ph.D.

University of California, Los Angeles

Stanley J. Huey, Jr., Ph.D.

University of Southern California

Bruce F. Chorpita, Ph.D.

University of California, Los Angeles 


\begin{abstract}
Author note
Correspondence concerning this manuscript should be addressed to Alayna Park, Ph.D., Department of Psychology, Palo Alto University, 1791 Arastradero Road, Palo Alto, CA 94304.

Email: apark1@paloaltou.edu. Twitter: @ ALParkPhD
\end{abstract}

Funding statement: The authors received no specific funding for this work.

Disclosure statement: Dr. Chorpita is the President of PracticeWise, LLC, which manages the PracticeWise Evidence-Based Services (PWEBS) Literature Database and created the PracticeWise Clinical Coding System referenced in this study.

Data availability statement: The data that support the findings of this study are available from the corresponding author, AP, upon reasonable request. 


\begin{abstract}
This review summarized the literature on psychosocial interventions for youth of color. Ninetythree journal articles of randomized clinical trials, with samples comprised of youth of color, published between 1974 and 2018 were coded for sample characteristics, intervention characteristics, and strategies for incorporating culture into psychotherapy. Results found 69 psychosocial interventions to be efficacious for youth of color; $32 \%$ of these psychosocial interventions included a strategy for incorporating culture into psychotherapy. The evidence base was largest for Black and Hispanic/Latinx populations and for psychosocial interventions targeting disruptive behavior problems. The most common strategies for incorporating culture into treatment among effective psychosocial interventions were employing procedures for addressing cultural context and including providers with awareness and knowledge of the client's culture. The inclusion of strategies for incorporating culture was not associated with treatment efficacy. Findings from this review highlight the laudable efforts that have been made to identify efficacious psychosocial interventions for youth of color and illuminate remaining gaps in the evidence base (e.g., efficacious psychosocial interventions for Asian, Native American and Alaska Native, and Native Hawaiian and Pacific Islander youth). Findings also emphasize the nuance of providing effective mental health services that are compatible with client's cultural worldviews, values, and practices and allude to the promise of decision support tools to help providers determine whether, when, and how to culturally tailor their psychotherapy with youth of color.
\end{abstract}




\section{Strategies for incorporating culture into psychosocial interventions for youth of color}

The dissemination and widespread implementation of evidence-based mental health interventions (EBIs) has been a longstanding public health priority (Task Force on Promotion and Dissemination of Psychological Procedures, 1995). As a result of laudable efforts to develop effective psychotherapy practices, there are now more than 800 EBI protocols for addressing a variety of child and adolescent mental health problems (e.g., Coping Cat for child anxiety; Kendall et al., 1990) (PracticeWise, 2020). However, there are only 4 well-established and 10 probably efficacious or possibly efficacious psychosocial intervention approaches for youth of color (e.g., cognitive behavioral therapy for Hispanic/Latinx youth with anxiety; Pina et al., 2019). The lack of representation of youth of color in randomized clinical trials has caused many to justifiably question the fit between EBIs and the racially and ethnically diverse youth and families who seek mental health services (Reding et al., 2018).

Scholars have proposed several avenues for filling this disconcerting knowledge gap. A consistent first-line recommendation has been to make concerted efforts toward recruiting youth of color in randomized clinical trials (Huey \& Polo, 2008; Miranda, 1996; Polo et al., 2019; Sugden \& Moulson, 2015). Improved representation can facilitate the identification of EBIs for youth of color, as well as expand current knowledge about the robustness of EBIs for addressing youth mental health problems. However, as evidenced by the incrementally increasing number of EBIs for youth of color - from 0 EBIs in 1996 (Chambless, 1996) to 14 in 2019 (Pina et al., 2019) - testing EBIs requires significant time, efforts, and resources. Additionally, EBIs are not culturally-neutral, and it would be a disservice not to attend to cultural factors ${ }^{1}$ that may impact psychotherapy engagement and response (Meyer $\&$ Zane, 2013; Sorenson \& Harrell, 2021).

\footnotetext{
${ }^{1}$ Whaley and Davis (2007) define culture as "a dynamic process involving worldviews and ways of living in a physical and social environment shared by groups, which are passed from generation to generation and may be modified by contacts between cultures in a particular social, historical, and political context" (p. 564). Although this systematic review focuses on racial and ethnic groups, we use the term culture when referring to worldviews, values, and practices shared by groups that are not solely identified by race and ethnicity (e.g., language, nationality).
} 
A complementary approach for extending the reach of EBIs is to develop and test culturally tailored ${ }^{2}$ EBIs, or EBIs that have been systemically modified to be more compatible with the client's cultural worldviews, values, and practices (Bernal et al., 2009). Several scholars have proposed cultural tailoring models for better meeting the mental health needs of youth of color. For example, Bernal and colleagues' (1995) widely used ecological validity model outlines eight dimensions for guiding cultural tailoring of EBIs: (1) language (i.e., culturally appropriate and syntonic language); (2) persons (i.e., racial and ethnic similarities between the mental health provider and their client); (3) metaphors (i.e., symbols and concepts shared by the cultural group); (4) content (i.e., knowledge of cultural values and traditions); (5) concepts (i.e., intervention concepts consonant with the cultural group); (6) goals (i.e., framing of psychotherapy goals within cultural values); (7) methods (i.e., framing of treatment procedures within cultural values); and (8) context (i.e., consideration of cultural context, such as acculturative stress, during assessment and intervention). As another example, Lau (2006) proposed a selective and directed cultural tailoring model for using evidence to selectively identify when cultural tailoring of EBIs may be most indicated and to direct how to culturally tailor EBIs to improve fit for the target cultural group. Studies have found that culturally tailored EBIs are efficacious for youth of color (Benish et al., 2011; Lau et al., 2011). However, findings on the relative efficacy of culturally tailored EBIs compared with unmodified EBIs are inconclusive (Huey et al., 2014). Additionally, developing and testing culturally tailored EBIs encounters the aforementioned issue of being time, effort, and resource intensive.

One solution that leverages the extant evidence base to enhance the generalizability of EBIs is to distill common elements from EBIs. Chorpita and colleagues' (2005) proposed a distillation model that involves extracting information about elements commonly featured in EBIs. This distillation model has been applied to characterize common elements of youth psychosocial interventions (e.g., Chorpita et al., 2011), interventions for promoting treatment engagement (e.g., Becker et al., 2018), and mental health

\footnotetext{
${ }^{2}$ The terms culturally tailored, culturally adapted, culturally competent, culturally responsive, and culturally sensitive have somewhat different meanings but tend to be used interchangeably by scholars (Huey et al., 2014). In this paper, we use the first term because cultural tailoring refers a more purposeful process of incorporating cultural factors into interventions.
} 
promotion programs (e.g., Boustani et al., 2015; 2020). Using a distillation model to characterize common strategies for incorporating culture into psychotherapy could increase the applicability of the current evidence base by generating a library of promising ideas for culturally tailoring interventions. These self-contained strategies for incorporating culture into psychotherapy could then theoretically be integrated into any intervention (e.g., adding a cultural tailoring strategy into existing EBIs) - thereby alleviating the burden involved in developing separate culturally tailored intervention protocols (e.g., developing culturally tailored intervention protocols for Hispanic/Latinx youth with anxiety, Black youth with anxiety, and so forth).

The current study reviewed the literature on psychosocial interventions for youth of color, specifically aiming to: (1) characterize efficacious psychosocial interventions for youth of color (e.g., treatment family, setting, format, target problem); (2) examine the association between the cultural tailoring and efficacy of youth psychosocial interventions; and (3) distill common strategies for incorporating culture into psychotherapy for youth of color. Through summarizing current knowledge about psychosocial interventions for youth of color, we hoped to highlight areas in the mental health services literature that could benefit from further study and to identify promising strategies for directing how to culturally tailor psychotherapy.

\section{Method}

\section{Search and Selection Criteria}

Potential journal articles were identified using the PracticeWise Evidence-Based Services

(PWEBS) Literature Database. The PWEBS Database includes studies of psychosocial interventions from 1965 to 2019 that were found through: (a) literature searches using electronic databases (e.g., PsycINFO, SocIndex, PubMed); (b) reviews of articles featured in meta-analyses; (c) examinations of EBI databases (e.g., National Registry of Evidence-Based Programs and Practices, Cochran Reviews, Campbell Collaboration); (d) surveys of the table of contents of recently released journal issues; and (e) nominations from experts in children's mental health. To be included in the PWEBS Database, articles have to: (a) describe a randomized clinical trial; (b) test at least one psychosocial intervention targeting a 
mental health problem, such as anxiety, attention problems or hyperactivity, autism, depression, disruptive behavior, eating problems, elimination problems, mania, substance use, suicidality, or traumatic stress; (c) have a youth sample with a mean age of 21 years or less; and (d) include youth presenting with or at-risk for a particular mental health problem. As of October 2020, there were 1,197 articles included in the PWEBS Database.

All studies from the PWEBS Database were screened for inclusion in the present review. Studies were required to have samples where at least $75 \%$ of participants identified as youth of color. This threshold is consistent with other reviews of psychosocial interventions for youth of color (e.g., Pina et al., 2019). Youth of color were defined as children or adolescents who identified as: (a) Black; (b) American Indian or Alaska Native; (c) Asian; (d) Hispanic/Latinx; (e) multiracial; or (f) Native Hawaiian or other Pacific Islander. Studies conducted outside of North America were not included in this review, as the personal and psychotherapy experiences of individuals from these racial and ethnic groups differ in other parts of the world (e.g., Saleem et al., 2020).

\section{Sample and Intervention Characteristics}

All studies in the PWEBS Database are coded using the PracticeWise Clinical Coding System (PracticeWise, 2012), which characterizes information related to study design, sample characteristics (i.e., race/ethnicity ${ }^{3}$, age, gender), intervention characteristics [i.e., type of study arm (e.g., active treatment, waitlist); treatment family (e.g., cognitive behavior therapy, parent management training); setting (e.g., school, clinic, home); format (e.g., youth individual, youth group, caregiver individual, caregiver group); provider education level (e.g., Master's level, doctoral level, graduate student); treatment target (e.g., disruptive behavior, depression, anxiety)], and clinical outcomes. Each study is coded by two coders who

\footnotetext{
${ }^{3}$ Studies were coded for the percentage of participants who identified as White, Black or African American, Hispanic or Latinx, Native American or Alaska Native, Asian, Native Hawaiian or Other Pacific Islander, multiracial, or "other" race. Notably, race and ethnicity are not uniformly reported in the mental health services literature. Accordingly, race and ethnicity were coded to the best of the coder's ability based on the information available in the study. For example, participants who identified as Hispanic may have been coded as Hispanic or Latinx, White, or other depending on how the authors of the journal article categorized race and ethnicity (e.g., Pimentel \& Balzhiser, 2012).
} 
have received extensive training in the coding system and who use a detailed coding manual. Inter-rater reliability among coders using this system has been reported to range from moderate to perfect $(\kappa=.66$ 1.00; Chorpita \& Daleiden, 2009). Any discrepancies between coders are resolved by an expert reviewer who also inspects all data for accuracy.

\section{"Winning" Status}

Clinical outcomes associated with each study arm were extracted from the PWEBS Database. Study arms that produced significantly better clinical outcomes than one or more study arms - as indicated by a Group x Time interaction or a between-group difference observed at the post-treatment assessment - were classified as "winning" study arms. "Wins" were chosen as an indicator of treatment effectiveness for this review because: (a) they signify greater efficacy relative to another study arm, without requiring homogeneity among comparison study arms; and (b) they are the primary measure of effectiveness used in studies employing the distillation model (e.g., Becker et al., 2018; Chorpita et al., 2011; Rith-Najarian et al., 2019).

\section{Strategies for Incorporating Culture}

Each active treatment study arm (i.e., study arm testing a psychosocial intervention or concurrent psychosocial and psychopharmacological treatments) was also coded for strategies for incorporating culture using a separate coding system developed by the first author. An intervention was operationalized as incorporating culture if it featured at least one element that was explicitly tailored for a specific racial or ethnic group. For example, an intervention that featured "role-plays... developed by and for urban African American girls in partnership with researchers" (Leff et al., 2015) would be considered to be culturally tailored, but an intervention that mentioned role-plays without describing the cultural relevance would not.

Based on a comprehensive literature review of cultural tailoring models and culturally tailored interventions as well as consultation with experts in culture and mental health, we generated a set of 40 initial codes of strategies for incorporating culture into psychotherapy. We then organized codes into overarching themes of intervention content (i.e., what providers work on with clients) and intervention 
process (i.e., how providers work with clients). Intervention content and intervention process are commonly used to characterize elements featured in psychosocial interventions (e.g., Garland et al., 2010; Stirman et al., 2019). These two overarching themes were further specified into six subthemes (see Table 1 for examples of each subtheme). Three subthemes related to intervention content: conceptualization (i.e., how information was framed), message (i.e., what was said by providers), and procedures (i.e., what providers did with clients). The other three subthemes related to intervention process: therapeutic style (i.e., how providers behave with clients), communication (i.e., how providers deliver the message), and change agents (i.e., persons involved in the intervention). These subthemes reflect multiple models of cultural tailoring. For example, the change agent subtheme is reminiscent of the persons dimension from Bernal and colleagues' (1995) ecological validity model but includes consultation with stakeholders from Sorenson and Harrell's (2021) 4-Domain Cultural Adaptation Model, training of providers in cultural awareness and knowledge from skills-based models (Huey et al., 2014), and collateral participants who are thought to be particularly integral to effective psychotherapy for some cultures (e.g., McCabe et al., 2005). Each subtheme initially contained 3-8 codes.

Three doctoral students were trained by the first author to apply themes, subthemes, and codes characterizing strategies for incorporating culture into psychotherapy. Each doctoral student then independently coded one-third of the studies. All studies were independently double-coded by the first author. The doctoral student coders and first author met monthly to discuss and refine the coding system. Codes that demonstrated poor inter-rater reliability were redefined, and codes that were assigned with low frequency were subsumed under a code with a broader definition. For instance, the initial change agent codes of consultation with experts, consultation with paraprofessionals, and consultation with stakeholders were subsumed under a broader final code of consultation. Inter-rater reliability was calculated using intraclass correlations based on a 2-way random effects model with measures of absolute agreement, using the mean of multiple raters. Table 2 displays a list of the final code names, definitions, and intraclass correlations representing inter-rater reliability. 


\section{Results}

This review included 93 studies testing psychosocial interventions with youth of color. Studies were published between 1974 and 2018 and included a total of 9,815 youth participants (range: 9-514 participants per study). Each study compared between two to six study arms $(M=2.37, S D=.79)$, for a total of 220 study arms. Study arms tested 167 psychosocial interventions (76\%), 30 waitlist or no treatment controls (14\%), 21 attentional controls (10\%; e.g., reading wellness articles), and 2 concurrent psychosocial and psychopharmacological treatments.

\section{Winning Intervention Characteristics}

Of the 169 study arms that tested a psychosocial intervention or concurrent psychosocial and psychopharmacological treatments, 69 interventions (41\%) outperformed a comparison study arm and thereby achieved "winning" status. Twenty-seven of these winning interventions (39\%) were tested with racially and ethnically diverse youth participants (i.e., sample included predominantly youth of color, with no single racial or ethnic minority group making up the majority of the sample); 21 winning interventions (30\%) were tested with youth participants who predominantly identified as Black; 17 winning interventions $(25 \%)$ were tested with youth participants who predominantly identified as Hispanic/Latinx; 2 winning interventions (3\%) were tested with youth participants who predominantly identified as Asian; and 2 winning interventions (3\%) were tested with youth participants who predominantly identified as Native American or Alaska Native. Figure 1 shows the number of winning interventions compared with the total number of interventions tested by predominant race of sample.

Winning interventions included cognitive behavior therapy $(n=16)$, parent management training $(n=8)$, family therapy $(n=8)$, motivational interviewing $(n=6)$, anger management $(n=5)$, interpersonal therapy $(n=3)$, play therapy $(n=3)$, social skills training $(n=3)$, attachment-based therapy $(n=2)$, mindfulness $(n=2)$, multisystemic therapy $(n=2)$, and a variety of other treatments (e.g., case management, cultural storytelling; $n=11)$. These interventions were delivered in schools $(n=32)$, clinics $(n=16)$, the community $(n=10)$, homes $(n=10)$, group homes $(n=2)$, and correctional facilities $(n=2)$.

The format of winning interventions involved individual therapy with youth $(n=25)$, group therapy with 
youth $(n=19)$, family therapy $(n=12)$, individual therapy with youth and caregivers $(n=8)$, individual therapy with caregivers $(n=7)$, group therapy with caregivers $(n=5)$, self-administration of the intervention $(n=4)$, and "other" formats $(n=10)$. These interventions were delivered by Master's level providers $(n=25)$, doctoral level providers $(n=17)$, graduate students $(n=15)$, teachers or school staff $(n$ $=4$ ), and various other providers (e.g., peers, Bachelor's level providers; $n=10$ ). Winning interventions demonstrated efficacy in addressing problems related to disruptive behavior $(n=25)$, substance use $(n=$ $8)$, anxiety $(n=8)$, traumatic stress, including child maltreatment $(n=5)$, transdiagnostic mechanisms (i.e., problems that cut across diagnostic boundaries) $(n=5)$, depression $(n=5)$, inattention or hyperactivity $(n=3)$, autism spectrum disorder $(n=1)$, and other problems (e.g., poor treatment engagement, crises, low self-efficacy; $n=6$ ) (Table 3).

\section{Cultural Tailoring of Psychosocial Interventions}

Of the 69 interventions that achieved winning status, 22 interventions (32\%) were culturally tailored. Ten of the 17 winning interventions (59\%) tested with Hispanic/Latinx youth were culturally tailored; 6 of the 21 winning interventions (29\%) tested with Black youth were culturally tailored; 4 of the 27 winning interventions (15\%) tested with racially and ethnically diverse youth were culturally tailored; and 2 of the 2 winning interventions (100\%) tested with Native American and Alaska Native youth were culturally tailored.

To present these results from another perspective, $45 \%$ of culturally tailored interventions achieved winning status; $67 \%$ of interventions tailored for Native American and Alaska Native youth achieved winning status; $50 \%$ of interventions tailored for Black youth achieved winning status; $45 \%$ of interventions tailored for Hispanic/Latinx youth achieved winning status; and 33\% of interventions tailored for racially and ethnically diverse youth achieved winning status (Table 4). Cultural tailoring was not associated with winning status, $\chi^{2}(1)=.47, p=.49$.

\section{Strategies for Incorporating Culture}

Five of the 12 (42\%) interventions that tailored the conceptualization achieved winning status; 6 of the $8(75 \%)$ interventions that tailored the message achieved winning status; 9 of the $20(45 \%)$ 
interventions that tailored the procedures achieved winning status; 3 of the $9(33 \%)$ interventions that tailored therapeutic style achieved winning status; 9 of the $21(43 \%)$ interventions that tailored the communication achieved winning status; and 16 of the 35 (46\%) interventions that tailored the change agent achieved winning status (see Table 2). Tailoring the conceptualization $\left(\chi^{2}(1)=.03, p=.87\right.$ ), message $\left(\chi^{2}(1)=3.51, p=.06\right)$, procedures $\left(\chi^{2}(1)=.76, p=.38\right)$, therapeutic style $\left(\chi^{2}(1)=.11, p=.75\right)$, communication $\left(\chi^{2}(1)=.04, p=.85\right)$, and change agent $\left(\chi^{2}(1)=.51, p=.48\right)$ were not associated with winning status ${ }^{4}$.

Across these subthemes of cultural tailoring strategies, the most common specific cultural tailoring strategies found in winning interventions were: employing procedures for addressing cultural context (procedures subtheme; $n=7$ ); matching problems with clients with similar cultural worldviews or training providers on the worldviews of other cultures (change agent subtheme; $n=7$ ); including collateral members (e.g., family members, teachers, peers) in intervention delivery (change agent subtheme; $n=6$ ); using culturally tailored materials (e.g., materials translated into the client's preferred language) (communication subtheme; $n=6$ ); employing procedures for increasing client engagement (procedures subtheme; $n=5$ ); and consulting with experts or stakeholders in designing the intervention (change agent subtheme; $n=5$ ) (see Table 2).

Winning, culturally tailored interventions included an average of 3.36 specific strategies $(S D=$ 2.90). Culturally tailored interventions that did not achieve winning status included an average of 2.96 specific strategies $(S D=2.36)$. The number of specific strategies for incorporating culture employed by interventions that did and did not achieve winning status was not significantly different, $t(47)=-.53, p=$ .60 .

\footnotetext{
${ }^{4}$ The same pattern of results was found when using a logistic regression with any cultural tailoring, tailored conceptualization, tailored message, tailored procedures, tailored therapeutic style, tailored communication, and tailored change agent predicting winning status.
} 


\section{Discussion}

This review summarized characteristics of efficacious psychosocial interventions for youth of color, including common strategies for incorporating culture into psychotherapy. By reviewing the literature on EBIs for youth of color, we hoped to identify promising psychotherapy elements for youth of color, as well as areas in the mental health services literature that warrant further study.

This review identified 93 randomized clinical trials that tested psychosocial interventions with youth of color. Across these studies, 69 interventions demonstrated superior clinical outcomes to a comparison study arm. The evidence base on efficacious psychosocial interventions was comprised largely of racially and ethnically diverse, Black, and Hispanic/Latinx youth samples. Only two winning study arms included predominantly Asian youth, and two winning study arms included predominantly Native American or Alaska Native youth; no studies included predominantly Native Hawaiian or other Pacific Islander youth. Results also showed that this evidence base was largest for the treatment of disruptive behavior problems, with limited EBI protocols for addressing substance use, anxiety, traumatic stress, and depression for youth of color. This glimpse into the evidence base points to fruitful areas for further investigation. For instance, concerted efforts must to made to recruit more Asian, Native Hawaiian and Pacific Islander, and Native American and Alaska Native youth into clinical trials testing psychosocial interventions to ensure that there are effective psychotherapy options for the entire serviceseeking population. Additionally, there remains a pressing need to identify EBIs, validated with historically underrepresented racial and ethnic groups, for many common youth mental health problems. As one example, consistent with other reviews (Pina et al., 2019), this study found zero EBIs for Black youth with depression or substance use problems. Such findings highlight the need to conscientiously recruit representative samples and to innovate solutions for providing effective psychotherapy for youth of color in the absence of research evidence.

This study also found that only one-third of the psychosocial interventions that demonstrated efficacy with youth of color incorporated culture. Additionally, less than half of the identified culturally tailored psychosocial interventions outperformed comparison study arms. These mixed findings on the 
efficacy of cultural tailoring are consistent with other reviews of EBIs for youth of color (Huey et al., 2014) and reflect the complexity of developing and implementing effective psychosocial interventions that are compatible with diverse clients' worldviews, values, and practices. The number of culturally tailored psychosocial interventions that demonstrated efficacy for youth of color is not inconsequential and suggest that there is value to incorporating culture into psychotherapy, as suggested by some metaanalyses (Nagayama Hall et al., 2016). At the same time, the lack of a clear association between cultural tailoring and superior client outcomes indicates remaining knowledge gaps in selecting and directing when and how to culturally tailor psychotherapy to optimize its effectiveness (e.g., Lau, 2006).

To direct how culture could be incorporated into psychotherapy, this review distilled cultural tailoring strategies that were featured in efficacious psychosocial interventions for youth of color. Culturally tailored EBIs employed a variety of strategies - the most common involving the use of procedures for addressing cultural context and the inclusion of providers with awareness and knowledge of the client's culture. Strategies for incorporating culture into psychotherapy were included in psychosocial interventions tested with racially and ethnically diverse, Black, Hispanic/Latinx, and Native American samples across various settings (i.e., school, clinic, home, community), using different formats (i.e., youth individual, youth group, family, youth and caregiver, caregiver individual, caregiver group, self-administration), and targeting a variety of mental health concerns (i.e., disruptive behavior, substance use, anxiety, depression, transdiagnostic mechanisms, traumatic stress, and inattention). These findings suggest that these cultural tailoring strategies are not protocol-specific but may be relatively selfcontained and could thereby theoretically be applied to any EBI protocol. Although this study lends insights into how to direct cultural tailoring, it is equally important to consider when to selectively cultural tailor psychotherapy. Results from this study hint at some cases where cultural tailoring may be most indicated; however, further research is needed to inform clear recommendations for selecting when to culturally tailor EBIs and then directing which cultural tailoring strategies to use given client and contextual factors. 
This review has several strengths, including its summary of 93 studies testing psychosocial interventions with youth of color and its identification of strategies for incorporating culture into psychotherapy; however, there are some limitations. One limitation is that the sample characteristics, intervention characteristics, and strategies for incorporating culture into psychotherapy were coded from journal articles of randomized clinical trials, and the information presented in this review is, thus, limited to the information reported in those articles. For instance, it is possible that some psychosocial interventions may have featured strategies for incorporating culture into psychotherapy that were not explicitly described in the article (see Huey \& Polo, 2008) and, accordingly, may not be reflected in this review. Relatedly, several journal articles referred readers to manuals for additional information about the psychosocial interventions under investigation. Perhaps unsurprisingly, manuals often have more comprehensive descriptions of interventions than journal articles (Knudsen et al., 2018); however, coding intervention manuals was outside of the scope of this review. As such, the identified intervention characteristics and strategies for incorporating culture into psychotherapy likely reflect the most notable intervention design features but may not reflect all aspects of the intervention design. On a related note, the often succinct descriptions of cultural tailoring and relative obscurity around intervention design decisions (e.g., whether the intervention was delivered in a school setting for cultural or other reasons) occasionally proved challenging for coding strategies for incorporating culture into psychotherapy. Interrater reliability was poor for 6 of the 20 cultural tailoring strategy codes; however, it should be noted that all final code applications were determined through consensus. Another limitation is that relatively few interventions featured strategies for incorporating culture into psychotherapy. For example, strategies for tailoring therapeutic style were featured in only nine interventions and were associated with only three efficacious interventions. Therapeutic style has been deemed by providers to be influential in providing effective psychotherapy for youth of color (Park, Boustani et al., 2020); yet, given the current evidence base, it is difficult to discern whether this type of cultural tailoring strategy is understudied, underreported, ineffective, or a combination of the above. Additionally, some cultural tailoring strategies 
(e.g., racial socialization) have not yet been tested in randomized clinical trials and, consequently, were not distilled in this review.

\section{Future Directions}

The current evidence base reflects diligent efforts in the development and testing of psychosocial interventions for youth of color. However, continued, concerted efforts are needed to fill current gaps in the mental health services literature and to demystify effective psychosocial intervention options for youth of color.

Specifically, proposals for future mental health services research should include plans for recruiting more representative samples. There is a particular need for studies including Asian, Native Hawaiian and Pacific Islander, and Native American or Alaska Native youth, as youth from these racial and ethnic groups make up a sizeable proportion of the service-seeking population but are largely absent from the extant literature. Additionally, studies investigating cultural tailoring should clearly define, operationalize, and describe cultural tailoring, as the use of broad language (e.g., "increasing compatibility with cultural worldviews") may contribute to the mixed findings on the effectiveness of culturally tailoring EBIs for youth of color. Furthermore, given that there are considerable racial and ethnic disparities in mental health care, future research must investigate not only selective and directed strategies for improving the quality and effectiveness of mental health services for youth of color but also strategies for increasing access to mental health services for youth of color (Alegria et al., 2010).

Future research should also continue to identify factors that may mediate or moderate the benefits of cultural tailoring, explore effective cultural tailoring strategies, and determine whether, when, and how to culturally tailor psychotherapy to best meet the needs for historically underrepresented racial and ethnic groups. A steadily growing number of psychosocial interventions feature selective and directed cultural tailoring (Fung et al., 2016; Ginsburg \& Drake, 2002; Huey \& Rank, 1984; Lau et al., 2011; Matos et al., 2009; Pina et al., 2012, Rosselló \& Bernal, 1999; Santisteban et al., 2011; Silverman et al., 1999; Young et al., 2010); however, many of these interventions are designed prior to implementation. As such, these interventions can address group-specific concerns, but they may not be designed to handle client-specific 
concerns or intragroup differences that may require the intervention to be adapted in real-time (Chorpita \& Daleiden, 2014; Nagayama Hall et al., 2021). A promising future research direction may, therefore, be to study and build decision support tools for helping mental health providers make real-time decisions about when and how to culturally tailor psychotherapy. These decision support tools would need to help providers assess for opportunities ripe for cultural tailoring (e.g., when a client is at risk for low engagement or is not responding as expected to an EBI) and select evidence-informed solutions (Park, Becker et al., 2020). As such, more research is needed to explicate client and contextual factors that call for cultural tailoring, identify effective cultural tailoring strategies, and map client and contextual factors with corresponding cultural tailoring strategies (e.g., assigning clients with low English proficiency to providers with appropriate language skills; focusing on engaging clients with personal or historical negative experiences with the healthcare system).

\section{Conclusion}

This review highlights the laudable work conducted by mental health services researchers to identify EBIs for youth of color, as well as the continued work that must be completed to increase representation within the evidence base. Findings underscore the complexity of designing and implementing effective and culturally compatible psychotherapy, particularly when considering the individual differences that exist among youth, families, providers, clinics, and mental health service systems. Fortunately, calls for mitigating racial and ethnic mental health disparities are gaining increasing traction - and with aligned research, practice, policy, and social initiatives, we can move closer to mental health parity. 


\section{References}

Alegria, M., Vallas, M., \& Pumariega, A. J. (2010). Racial and ethnic disparities in pediatric mental health. Child and Adolescent Psychiatric Clinics of North America, 19(4), 759-774. doi: 10.1016/j.chc.2010.07.001

Becker, K. D., Boustani, M., Gellatly, R., \& Chorpita, B. F. (2018). Forty years of engagement research in children's mental health services: Multidimensional measurement and practice elements. Journal of Clinical Child \& Adolescent Psychology, 47(1), 1-23. https://doi.org/10.1080/15374416.2017.1326121

Benish, S. G., Quintana, S., \& Wampold, B. E. (2011). Culturally adapted psychotherapy and the legitimacy of myth: A direct-comparison meta-analysis. Journal of Counseling Psychology, 58(3), 279-289. doi: 10.1037/a0023626

Bernal, G., Bonilla, J., \& Bellido, C. (1995). Ecological validity and cultural sensitivity for outcome research: Issues for the cultural adaptation and development of psychosocial treatments with Hispanics. Journal of Abnormal Child Psychology, 23(1), 67-82. doi: 10.1007/BF01447045

Bernal, G., Jiménez-Chafey, M. I., \& Domenech Rodríguez, M. M. (2009). Cultural adaptation of treatments: A resource for considering culture in evidence-based practice. Professional Psychology: Research and Practice, 40(4), 361-368. https://doi.org/10.1037/a0016401

Boustani, M. M., Frazier, S. L., Becker, K. D., Bechor, M., Dinizulu, S. M., Hedemann, E. R., Ogle, R. R., \& Pasalich, D. S. (2015). Common elements of adolescent prevention programs: Minimizing burden while maximizing reach. Administration and Policy in Mental Health and Mental Health Services Research, 42(2), 209-219. doi: 10.1007/s10488-014-0541-9

Boustani, M. M., Frazier, S. L., Chu, W., Lesperance, N., Becker, K. D., Helseth, S. A., Hedemann, E. R., Ogle, R. R., \& Chorpita, B. F. (2020). Common elements of childhood universal mental health programming. Administration and Policy in Mental Health and Mental Health Services Research, 47(3), 475-486. doi: 10.1007/s10488-020-01023-4 
Chambless, D. L. (1996). In defense of dissemination of empirically supported psychological interventions. Clinical Psychology: Science and Practice, 3(3), 230-235. http://dx.doi.org/10.1111/j.1468-2850.1996.tb00074.x

Chorpita, B. F., \& Daleiden, E. L. (2009). Mapping evidence-based treatments for children and adolescents: Application of the distillation and matching model to 615 treatments from 322 randomized trials. Journal of Consulting and Clinical Psychology, 77(3), 566-579. http://dx.doi.org/10.1037/a0014565

Chorpita, B. F., \& Daleiden, E. L. (2014). Structuring the collaboration of science and service in pursuit of a shared vision. Journal of Clinical Child \& Adolescent Psychology, 43(2), 323-338. https://doi.org/10.1080/15374416.2013.828297

Chorpita, B. F., Daleiden, E. L., Ebesutani, C., Young, J., Becker, K. D., Nakamura, B. J., . . Starace, N. (2011). Evidence-based treatments for children and adolescents: An updated review of indicators of efficacy and effectiveness. Clinical Psychology: Science and Practice, 18(2), 154-172. http://dx.doi.org/10.1111/j.1468-2850.2011.01247.x

Chorpita, B. F., Daleiden, E. L., \& Weisz, J. R. (2005). Identifying and selecting the common elements of evidence based interventions: A distillation and matching model. Mental Health Services Research, 7(1), 5-20. http://dx.doi.org/10.1007/s11020-005-1962-6

Costantino, G., \& Malgady, R. G. (1994). Storytelling through pictures: Culturally sensitive psychotherapy for Hispanic children and adolescents. Journal of Clinical Child Psychology, 23(1), 13-20. https://doi.org/10.1207/s15374424jccp2301_3

Fung, J., Guo, S., Jin, J., Bear, L., \& Lau, A. (2016). A pilot randomized trial evaluating a school-based mindfulness intervention for ethnic minority youth. Mindfulness, 7(4), 819-828. https://doi.org/10.1007/s12671-016-0519-7

Garland, A. F., Brookman-Frazee, L., Hurlburt, M. S., Accurso, E. C., Zoffness, R. J., Haine-Schlagel, R., \& Ganger, W. (2010). Mental health care for children with disruptive behavior problems: A view inside therapists' offices. Psychiatric Services, 61(8), 788-795. doi: 10.1176/ps.2010.61.8.788 
Garza, Y., \& Bratton, S. C. (2005). School-based child-centered play therapy with Hispanic children: Outcomes and cultural consideration. International Journal of Play Therapy, 14(1), 51-80. https://doi.org/10.1037/h0088896

Ginsburg, G. S., \& Drake, K. L. (2002). School-based treatment for anxious African-American adolescents: A controlled pilot study. Journal of the American Academy of Child \& Adolescent Psychiatry, 41(7), 768-775. doi: 10.1097/00004583-200207000-00007

Huey, W. C., \& Rank, R. C. (1984). Effects of counselor and peer-led group assertive training on black adolescent aggression. Journal of Counseling Psychology, 31(1), 95-98. https://doi.org/10.1037/0022-0167.31.1.95

Huey Jr, S. J., \& Polo, A. J. (2008). Evidence-based psychosocial treatments for ethnic minority youth. Journal of Clinical Child \& Adolescent Psychology, 37(1), 262-301. doi: $10.1080 / 15374410701820174$

Huey, S. J., Jr., Tilley, J. L., Jones, E. O., \& Smith, C. A. (2014). The contribution of cultural competence to evidence-based care for ethnically diverse populations. Annual Review of Clinical Psychology, 10, 305-338. http://dx.doi.org/10.1146/annurev-clinpsy-032813-153729

Kendall, P. C. (1990). Coping cat manual. Ardmore, PA: Workbook.

Knudsen, K. S., Boustani, M.B., Chu W., Wesley, K., and Chorpita, B. F. (2018). do current publication standards reliably describe content of manualized universal prevention programs? Poster presentation at the Association for Psychological Sciences (APS), San Francisco, CA.

Lau, A. S. (2006). Making the case for selective and directed cultural adaptations of evidence-based treatments: Examples from parent training. Clinical Psychology: Science and Practice, 13(4), 295-310. http://dx.doi.org/10.1111/j.1468-2850.2006.00042.x

Lau, A. S., Fung, J. J., Ho, L. Y., Liu, L. L., \& Gudiño, O. G. (2011). Parent training with high-risk immigrant Chinese families: A pilot group randomized trial yielding practice-based evidence. Behavior Therapy, 42(3), 413-426. http://dx.doi.org/10.1016/j.beth.2010.11.001 
Leff, S. S., Paskewich, B. S., Waasdorp, T. E., Waanders, C., Bevans, K. B., \& Jawad, A. F. (2015). Friend to friend: A randomized trial for urban African American relationally aggressive girls. Psychology of Violence, 5(4), 433-443. https://doi.org/10.1037/a0039724.

Martinez Jr, C. R., \& Eddy, J. M. (2005). Effects of culturally adapted parent management training on Latino youth behavioral health outcomes. Journal of Consulting and Clinical Psychology, 73(5), 841-851. https://doi.org/10.1037/0022-006X.73.5.841

Matos, M., Bauermeister, J. J., \& Bernal, G. (2009). Parent-child interaction therapy for Puerto Rican preschool children with ADHD and behavior problems: A pilot efficacy study. Family Process, 48(2), 232-252. https://doi.org/10.1111/j.1545-5300.2009.01279.x

McCabe, K., \& Yeh, M. (2009). Parent-child interaction therapy for Mexican Americans: A randomized clinical trial. Journal of Clinical Child \& Adolescent Psychology, 38(5), 753-759. https://doi.org/10.1080/15374410903103544

McCabe, K. M., Yeh, M., Garland, A. F., Lau, A. S., \& Chavez, G. (2005). The GANA program: A tailoring approach to adapting parent child interaction therapy for Mexican Americans. Education and Treatment of Children, 28(2), 111-129.

McKay, M. M., Nudelman, R., McCadam, K., \& Gonzales, J. (1996). Evaluating a social work engagement approach to involving inner-city children and their families in mental health care. Research on Social Work Practice, 6(4), 462-472. https://doi.org/10.1177/104973159600600404

Meyer, O. L., \& Zane, N. (2013). The influence of race and ethnicity in client's experiences of mental health treatment. Journal of Community Psychology, 41(7), 884-901. doi: 10.1002/jcop.21580

Miranda, J. (1996). Introduction to the special section on recruiting and retaining minorities in psychotherapy research. Journal of Consulting and Clinical Psychology, 64(5), 848-850.

Nagayama Hall, G. C. N., Berkman, E. T., Zane, N. W., Leong, F. T., Hwang, W. C., Nezu, A. M., ... \& Huang, E. R. (2021). Reducing mental health disparities by increasing the personal relevance of interventions. American Psychologist, 76(1), 91-103. 103. https://doi.org/10.1037/amp0000616 
Nagayama Hall, G. C., Ibaraki, A. Y., Huang, E. R., Marti, C. N., \& Stice, E. (2016). A meta-analysis of cultural adaptations of psychological interventions. Behavior Therapy, 47(6), 993-1014. doi: 10.1016/j.beth.2016.09.005

Patten, C. A., Fadahunsi, O., Hanza, M. M., Smith, C. A., Decker, P. A., Boyer, R., ... \& Offord, K. P. (2014). Tobacco cessation treatment for Alaska Native adolescents: Group randomized pilot trial. Nicotine \& Tobacco Research, 16(6), 836-845. https://doi.org/10.1093/ntr/ntu004

Park, A. L., Becker, K. D., Boustani, M. M., \& Chorpita, B. F. (2020). Decision-making in mental health care: measuring provider and supervisor use of evidence. Administration and Policy in Mental Health and Mental Health Services Research, 47(3), 344-356. doi: 10.1007/s10488-019-00989-0

Park, A. L., Boustani, M. M., Saifan, D., Gellatly, R., Letamendi, A., Stanick, C., Regan, J., Perez, G., Manners, D., \& Reding, M. E. (2019). Community mental health professionals' perceptions about engaging underserved populations. Administration and Policy in Mental Health and Mental Health Services Research, 47(3), 366-379. doi: 10.1007/s10488-019-00994-3

Pimentel, C., \& Balzhiser, D. (2012). The double occupancy of Hispanics: Counting race and ethnicity in the US census. Journal of Business and Technical Communication, 26(3), 311-339. https://doi.org/10.1177/1050651912439696

Pina, A. A., Polo, A. J., \& Huey, S. J. (2019). Evidence-based psychosocial interventions for ethnic minority youth: The 10-year update. Journal of Clinical Child and Adolescent Psychology, 48(2), 179-202. http://dx.doi.org/10.1080/15374416.2019.1567350

Pina, A. A., Zerr, A. A., Villalta, I. K., \& Gonzales, N. A. (2012). Indicated prevention and early intervention for childhood anxiety: A randomized trial with Caucasian and Hispanic/Latino youth. Journal of Consulting and Clinical Psychology, 80(5), 940-946. doi: 10.1037/a0029460

Polo, A. J., Makol, B. A., Castro, A. S., Colón-Quintana, N., Wagstaff, A. E., \& Guo, S. (2019). Diversity in randomized clinical trials of depression: A 36-year review. Clinical Psychology Review, 67, 22-35. doi: 10.1016/j.cpr.2018.09.004

PracticeWise. (2012). Psychosocial and combined treatments coding manual. Satellite Beach, FL: Author. 
PracticeWise (2020). Evidence-based youth mental health services literature database. Retrieved from https://www.practicewise.com/pwebs_1/index.aspx

Reding, M. E., Guan, K., Regan, J., Palinkas, L. A., Lau, A. S., \& Chorpita, B. F. (2018). Implementation in a changing landscape: provider experiences during rapid scaling of use of evidence-based treatments. Cognitive and Behavioral Practice, 25(2), 185-198. https://doi.org/10.1016/j.cbpra.2017.05.005

Rith-Najarian, L., Mesri, B., Park, A. L., Sun, M., Chavira, D. A., \& Chorpita, B. F. (2019). Durability of cognitive behavioral therapy effects for youth and adolescents with anxiety, depression, or traumatic stress: A meta-analysis on long-term follow-ups. Behavior Therapy, 50(1), 225-240. http://dx.doi.org/10.1016/j.beth.2018.05.006

Rosselló, J., \& Bernal, G. (1999). The efficacy of cognitive-behavioral and interpersonal treatments for depression in Puerto Rican adolescents. Journal of Consulting and Clinical Psychology, 67(5), 734-745. doi: 10.1037//0022-006x.67.5.734

Saleem, F. T., Anderson, R. E., \& Williams, M. (2020). Addressing the "myth" of racial trauma: developmental and ecological considerations for youth of color. Clinical Child and Family Psychology Review, 23(1), 1-14. https://doi.org/10.1007/s10567-019-00304-1

Santisteban, D. A., Mena, M. P., \& McCabe, B. E. (2011). Preliminary results for an adaptive family treatment for drug abuse in Hispanic youth. Journal of Family Psychology, 25(4), 610-614. doi: $10.1037 / \mathrm{a} 0024016$

Schinke, S. P., Orlandi, M. A., Botvin, G. J., Gilchrist, L. D., Trimble, J. E., \& Locklear, V. S. (1988). Preventing substance abuse among American-Indian adolescents: A bicultural competence skills approach. Journal of Counseling Psychology, 35(1), 87-90. https://doi.org/10.1037/00220167.35.1.87

Sibley, M. H., Graziano, P. A., Kuriyan, A. B., Coxe, S., Pelham, W. E., Rodriguez, L., ... \& Ward, A. (2016). Parent-teen behavior therapy + motivational interviewing for adolescents with ADHD. 
Journal of Consulting and Clinical Psychology, 84(8), 699-712.

https://doi.org/10.1037/ccp0000106

Silverman, W. K., Kurtines, W. M., Ginsburg, G. S., Weems, C. F., Lumpkin, P. W., \& Carmichael, D. H. (1999). Treating anxiety disorders in children with group cognitive-behavioral therapy: A randomized clinical trial. Journal of Consulting and Clinical Psychology, 67(6), 995-1003. doi: 10.1037//0022-006x.67.6.995

Sorenson, C., \& Harrell, S. P. (2021). Development and testing of the 4-Domain Cultural Adaptation Model (CAM4). Professional Psychology: Research and Practice, 52(3), 250-259. https://doi.org/10.1037/pro0000370

Stirman, S. W., Baumann, A. A., \& Miller, C. J. (2019). The FRAME: an expanded framework for reporting adaptations and modifications to evidence-based interventions. Implementation Science, 14(1), 1-10. https://doi.org/10.1186/s13012-019-0898-y

Sugden, N. A., \& Moulson, M. C. (2015). Recruitment strategies should not be randomly selected: empirically improving recruitment success and diversity in developmental psychology research. Frontiers in Psychology, 6, 523. 10.3389/fpsyg.2015.00523

Szapocznik, J., Rio, A., Murray, E., Cohen, R., Scopetta, M., Rivas-Vazquez, A., . . Kurtines, W. (1989). Structural family versus psychodynamic child therapy for problematic Hispanic boys. Journal of Consulting and Clinical Psychology, 57(5), 571-578. http://dx.doi.org/10.1037/0022006X.57.5.571

Task Force on Promotion and Dissemination of Psychological Procedures (1995). A reported adopted by the Division 12 board - October 1993. Retrieved from http://www.div12.org/sites/default/files/InitialReportOfTheChamblessTaskForce.pdf

Whaley, A. L., \& Davis, K. E. (2007). Cultural competence and evidence-based practice in mental health services: A complementary perspective. American Psychologist, 62(6), 563-574. https://doi.org/10.1037/0003-066X.62.6.563 
Table 1

Examples of cultural tailoring strategies found in winning interventions.

\section{Cultural Tailoring Examples}

\section{Strategy}

Conceptualization • "The team considered the conceptual-theoretical relevance (e.g., 'Is the concept of contingent positive reinforcement to encourage prosocial behavior relevant or appropriate within this particular cultural frame?')... of each component...” (Martinez \& Eddy, 2005)

- "Cultural concepts were referenced throughout treatment so that the program could be presented in ways that were congruent with the parents' belief system." (McCabe \& Yeh, 2009)

- "We adapted a cognitive-behavioral and interpersonal treatment for depressed Puerto Rican adolescents, considering cultural, developmental, and socioeconomic factors and using a framework for ecological validity and culturally sensitive criteria.” (Rosselló \& Bernal, 1999)

Message $\quad$ • “...examples were modified to reflect the daily experiences and idiomatic expressions of Puerto Rican families.” (Matos et al., 2009)

- "Communication skills were introduced with biculturally relevant examples of verbal and nonverbal influences on substance use." (Schinke et al., 1988)

- "Other adaptations to the program included... framing program as an educational/skill building." (McCabe \& Yeh, 2009)

- "The team also identified new content areas to be developed to address the culturally specific risk and protective factors involved in adjustment outcomes for Latino parents and youth (e.g., family acculturation issues, structural barriers such as discrimination)." (Martinez \& Eddy, 2005)

- "Other adaptations to the program included... increasing orientation to therapy; increasing session time for rapport building... and implementing an engagement protocol.” (McCabe \& Yeh, 2009)

- "Differences in the race or ethnicity of the clinician and client were always raised." (McKay et al., 1998) 
Style

• "Parents were interviewed before and after the therapy in a climate of utmost respect." (Rosselló \& Bernal, 1999)

- "The foundation of a collaborative process between the client and the worker began during the first interview." (McKay et al., 1996)

Communication - "Selection of multicultural toys was decided by a panel of five Hispanic, Spanish-speaking, registered play therapists who conduct CCPT with Hispanic children in the Southwest United States." (Garza \& Bratton, 2005)

- "The experimental intervention consisted of a story-telling modality based on pictorial stimuli depicting Hispanic cultural elements (e.g., traditional foods, games, sex roles) and Hispanic families and neighborhoods (e.g., stores or 'bodegas') in urban settings.” (Costantino \& Malgady, 1994)

- "Videos, role-plays, and cartoons... were developed by and for urban African American girls in partnership with researchers." (Leff et al., 2015)

Change Agent $\quad$ • "Families were assigned to clinicians based upon preferred language (Spanish vs. English)...” (Sibley et al., 2016)

- “To enhance 'cultural acceptability,' elders and Alaska Native teen advisors provided intra-treatment support for quitting and shared their personal stories with participants in the form of talking circles." (Patten et al., 2014)

- "The facilitator should also be knowledgeable and comfortable with a transcultural perspective and familiar with the cultures involved." (Szapocznik et al., 1989) 
Table 2

Cultural Tailoring Strategies, Definitions, and Occurrences

\begin{tabular}{|c|c|c|c|c|c|}
\hline $\begin{array}{l}\text { Cultural Tailoring } \\
\text { Strategy Code }\end{array}$ & Definition & ICC & $\begin{array}{c}\text { Winning } \\
\text { Interventions }\end{array}$ & $\begin{array}{c}\text { Total } \\
\text { Interventions }\end{array}$ & $\%$ \\
\hline Conceptualization & How information was framed & .56 & 5 & 12 & $42 \%$ \\
\hline Concepts & Intervention concepts were framed within cultural values. & .46 & 4 & 11 & $36 \%$ \\
\hline Other & Conceptualization that does not fit with other categories (e.g., strengths-based). & .00 & 0 & 2 & $0 \%$ \\
\hline Message & What was said by provider & .71 & 6 & 8 & $75 \%$ \\
\hline Phrases & Message that involves culturally tailored label, saying, proverb, or example. & .72 & 4 & 4 & $100 \%$ \\
\hline Other & $\begin{array}{l}\text { Message that does not fit with other categories (e.g., intervention framed as } \\
\text { educational; providers referred to as "entenadores") }\end{array}$ & .85 & 3 & 5 & $60 \%$ \\
\hline Procedures & What providers asked clients to do & .77 & 9 & 20 & $45 \%$ \\
\hline Context & Procedure for addressing cultural context. & .79 & 7 & 15 & $47 \%$ \\
\hline Engagement & Procedure for increasing client engagement in the intervention. & 1.00 & 5 & 5 & $100 \%$ \\
\hline Personalization & Procedure that has been personalized for the client (e.g., modular intervention). & .88 & 3 & 5 & $60 \%$ \\
\hline Setting & $\begin{array}{l}\text { Intervention delivered in a modified setting than originally intended (e.g., } \\
\text { school, church). }\end{array}$ & -.02 & 2 & 5 & $40 \%$ \\
\hline Pacing & $\begin{array}{l}\text { Intervention delivered with modified pacing or timing than originally intended } \\
\text { (e.g., completed in more days). }\end{array}$ & .80 & 2 & 3 & $67 \%$ \\
\hline Other & $\begin{array}{l}\text { Procedure that does not fit with the other categories (e.g., connected families } \\
\text { with community resources) }\end{array}$ & -.02 & 2 & 4 & $50 \%$ \\
\hline Style & How provider behaves with client & .71 & 3 & 9 & $33 \%$ \\
\hline Collaborative & Style that emphasizes collaboration between provider and client. & .92 & 1 & 4 & $25 \%$ \\
\hline
\end{tabular}




\begin{tabular}{|c|c|c|c|c|c|}
\hline Respectful & Style that involves provider attending to issues of respect. & .92 & 2 & 3 & $67 \%$ \\
\hline Other & Style that does not fit with other categories (e.g., nonjudgmental stance). & .66 & 0 & 3 & $0 \%$ \\
\hline Communication & How provider delivers the message & .75 & 9 & 21 & $43 \%$ \\
\hline Materials & $\begin{array}{l}\text { Communication that involves culturally tailored materials, such as translated } \\
\text { materials or materials that include representations of the target ethnic/racial } \\
\text { group. }\end{array}$ & .91 & 6 & 14 & $43 \%$ \\
\hline Mode & Communication through a particular mode, such as storytelling or didactics. & .44 & 3 & 8 & $38 \%$ \\
\hline Other & Communication that does not fit with other categories. & -.04 & 3 & 6 & $50 \%$ \\
\hline Change Agent & Person(s) involved in the intervention & .82 & 16 & 35 & $46 \%$ \\
\hline Provider & $\begin{array}{l}\text { Provider was matched with clients with similar cultural worldviews, or received } \\
\text { training on the worldviews of other cultures. }\end{array}$ & .79 & 7 & 20 & $35 \%$ \\
\hline Consultation & Experts and/or stakeholders were consulted in designing the intervention. & .70 & 5 & 14 & $36 \%$ \\
\hline Collateral & $\begin{array}{l}\text { Family members, teachers, neighbors, and/or peers supported intervention } \\
\text { delivery. }\end{array}$ & 1.00 & 6 & 9 & $67 \%$ \\
\hline Other & Change agent that does not fit with other categories (e.g., interpreter). & .62 & 3 & 6 & $50 \%$ \\
\hline
\end{tabular}


Figure 1

Comparison of culturally tailored, winning, and total psychosocial interventions by predominant race/ethnicity of sample.

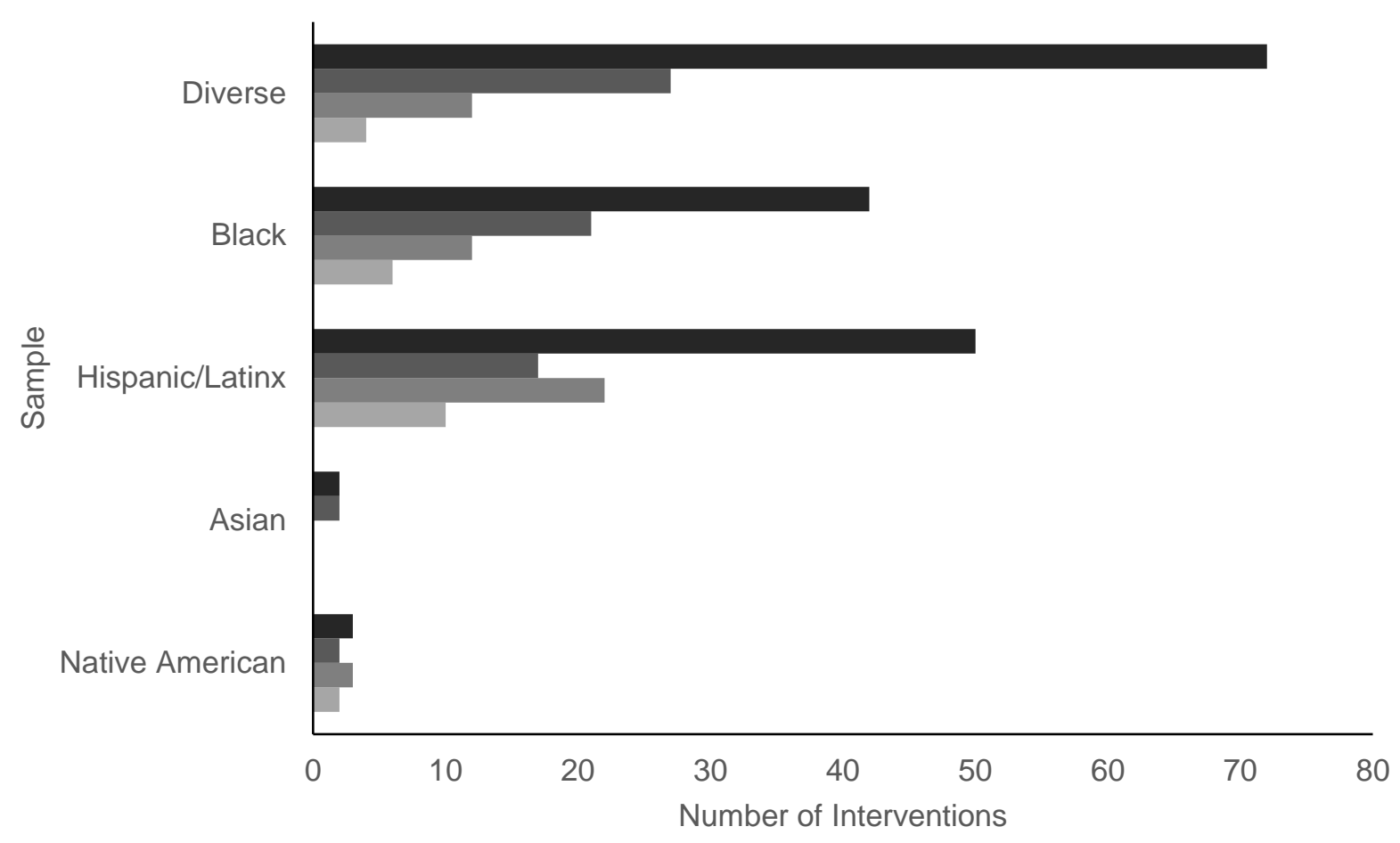

- Total Interventions

- Winning Interventions

- Culturally Tailored Interventions

- Winning Culturally Tailored Interventions 
Table 3

Number and percentage of winning interventions by race of sample and treatment characteristic.

\begin{tabular}{|c|c|c|c|c|c|c|c|c|c|c|c|c|c|c|c|c|c|c|}
\hline \multirow[t]{2}{*}{ Treatment Characteristic } & \multicolumn{3}{|c|}{ All Youth } & \multicolumn{3}{|c|}{ Diverse Sample } & \multicolumn{3}{|c|}{ Black Sample } & \multicolumn{3}{|c|}{$\begin{array}{c}\text { Hispanic/Latinx } \\
\text { Sample }\end{array}$} & \multicolumn{3}{|c|}{ Asian Sample } & \multicolumn{3}{|c|}{$\begin{array}{l}\text { Native } \\
\text { American } \\
\text { Sample }\end{array}$} \\
\hline & WCT & $\mathrm{W}$ & $\mathrm{T}$ & WCT & $\mathrm{W}$ & $\mathrm{T}$ & WCT & $\mathrm{W}$ & $\mathrm{T}$ & WCT & $\mathrm{W}$ & $\mathrm{T}$ & WCT & $\mathrm{W}$ & $\mathrm{T}$ & WCT & $\mathrm{W}$ & $\mathrm{T}$ \\
\hline \multicolumn{19}{|l|}{ Setting } \\
\hline School & 9 & 32 & 62 & 1 & 12 & 20 & 5 & 16 & 28 & 3 & 4 & 14 & -- & -- & -- & -- & -- & -- \\
\hline Clinic & 9 & 16 & 35 & 1 & 5 & 12 & 1 & 1 & 4 & 7 & 10 & 19 & -- & -- & -- & -- & -- & -- \\
\hline Home & 4 & 10 & 29 & 2 & 6 & 14 & 1 & 2 & 10 & 1 & 1 & 3 & 0 & 1 & 1 & 0 & 0 & 1 \\
\hline Community & 3 & 10 & 16 & 0 & 2 & 5 & 1 & 6 & 9 & 1 & 1 & 1 & -- & -- & -- & 1 & 1 & 1 \\
\hline Other & 3 & 16 & 59 & 1 & 9 & 34 & 0 & 1 & 6 & 1 & 4 & 17 & 0 & 1 & 1 & 1 & 1 & 1 \\
\hline \multicolumn{19}{|l|}{ Format } \\
\hline Youth individual & 5 & 25 & 61 & 2 & 13 & 34 & 0 & 7 & 16 & 3 & 5 & 11 & -- & -- & -- & -- & -- & -- \\
\hline Youth group & 8 & 25 & 53 & 0 & 6 & 17 & 5 & 15 & 23 & 1 & 2 & 11 & -- & -- & -- & 2 & 2 & 2 \\
\hline Family & 4 & 12 & 36 & 2 & 6 & 13 & 0 & 1 & 4 & 2 & 5 & 19 & -- & -- & -- & -- & -- & -- \\
\hline Caregiver individual & 2 & 7 & 21 & 1 & 3 & 8 & 1 & 2 & 7 & 0 & 1 & 4 & 0 & 1 & 1 & 0 & 0 & 1 \\
\hline Youth and caregiver & 2 & 8 & 20 & 0 & 4 & 11 & 0 & 1 & 3 & 2 & 2 & 5 & 0 & 1 & 1 & -- & -- & -- \\
\hline Caregiver group & 2 & 5 & 13 & 0 & 1 & 4 & 0 & 2 & 3 & 2 & 2 & 6 & -- & -- & -- & -- & -- & -- \\
\hline Self-administration & 2 & 4 & 4 & 1 & 1 & 1 & -- & -- & -- & -- & -- & -- & 0 & 2 & 2 & 1 & 1 & 1 \\
\hline
\end{tabular}




\section{Target Problem}

\begin{tabular}{|c|c|c|c|c|c|c|c|c|c|c|c|c|c|c|c|c|c|}
\hline Disruptive behavior & 10 & 25 & 51 & 3 & 10 & 24 & 4 & 10 & 18 & 3 & 5 & 9 & -- & -- & -- & -- & -- \\
\hline Substance use & 4 & 8 & 43 & 0 & 2 & 19 & 0 & 0 & 3 & 2 & 4 & 19 & -- & -- & -- & 2 & 2 \\
\hline Anxiety & 2 & 8 & 17 & -- & -- & -- & 1 & 7 & 9 & 1 & 1 & 8 & -- & -- & -- & -- & -- \\
\hline Traumatic stress & 0 & 5 & 15 & 0 & 6 & 10 & 0 & 2 & 6 & 0 & 0 & 1 & -- & -- & -- & -- & -- \\
\hline Transdiagnostic & 0 & 5 & 10 & 0 & 3 & 5 & 0 & 0 & 1 & 0 & 2 & 4 & -- & -- & -- & -- & -- \\
\hline Depression & 2 & 5 & 9 & 0 & 2 & 4 & -- & -- & -- & 2 & 3 & 5 & -- & -- & -- & -- & -- \\
\hline Inattention & 0 & 3 & 6 & -- & -- & -- & 0 & 1 & 2 & 2 & 2 & 4 & -- & -- & -- & -- & -- \\
\hline Autism spectrum disorder & 0 & 1 & 2 & 0 & 2 & 2 & -- & -- & -- & -- & -- & -- & -- & -- & -- & -- & -- \\
\hline Other & 2 & 6 & 14 & 1 & 3 & 8 & 1 & 1 & 3 & -- & -- & -- & 0 & 2 & 2 & 0 & 0 \\
\hline
\end{tabular}

Note: $\mathrm{WCT}=$ Number of winning culturally tailored interventions. $\mathrm{W}=$ Number of winning interventions. $\mathrm{T}=$ Total number of interventions. 
Table 4

Number and percentage of winning interventions by race/ethnicity of sample and cultural tailoring strategy.

\begin{tabular}{|c|c|c|c|c|c|c|c|c|}
\hline \multirow[b]{2}{*}{ Cultural Tailoring Strategy } & \multicolumn{2}{|c|}{ Diverse Sample } & \multicolumn{2}{|c|}{ Black Sample } & \multicolumn{2}{|c|}{ Hispanic/ Latinx Sample } & \multicolumn{2}{|c|}{ Native American Sample } \\
\hline & Winning & Total & Winning & Total & Winning & Total & Winning & Total \\
\hline Any strategy & 4 & 12 & 6 & 12 & 10 & 22 & 2 & 3 \\
\hline Conceptualization & -- & -- & 0 & 2 & 4 & 8 & 1 & 2 \\
\hline Message & -- & -- & 1 & 1 & 4 & 6 & 1 & 1 \\
\hline Procedures & 3 & 6 & 0 & 2 & 4 & 10 & 1 & 2 \\
\hline Style & 0 & 3 & 1 & 3 & 2 & 2 & 0 & 1 \\
\hline Communication & 3 & 6 & 2 & 3 & 4 & 11 & 0 & 1 \\
\hline Change agent & 2 & 6 & 2 & 6 & 10 & 20 & 2 & 3 \\
\hline
\end{tabular}

Note: The two study arms with predominantly Asian samples did not include any cultural tailoring strategies. 